\begin{tabular}{|c|c|c|}
\hline & Global Journal of Mathematical Analysis, 5(1) (2017) 1-5 & \\
\hline & Global Journal of Mathematical Analysis & \\
\hline SPC & $\begin{array}{c}\text { Website: www.sciencepubco.com/index.php/GJMA } \\
\text { doi: } 10.14419 / \text { gima.v5il.6949 } \\
\text { Research paper }\end{array}$ & \\
\hline
\end{tabular}

\title{
On Generalized k-Fibonacci Sequence by Two-Cross-Two Matrix
}

\author{
Arfat Ahmad Wani ${ }^{1 *}$, G.P.S. Rathore ${ }^{2}$ and Kiran Sisodiya ${ }^{3}$ \\ ${ }^{1}$ School of Studies in Mathematics, Vikram University, Ujjain, India \\ ${ }^{2}$ Department of Mathematics, Horticulture College, Mandsaur, India \\ ${ }^{3}$ School of Studies in Mathematics, Vikram University, Ujjain, India \\ *Corresponding author E-mail:arfatahmadwani@gmail.com
}

\begin{abstract}
In this study we define a new generalized k-Fibonacci sequence associated with its two cross two matrix called generating matrix. After use the matrix representation we find many interesting properties such as $n$th power of the matrix, Cassini's Identity of generalized k-Fibonacci sequence as well as Binet's formula for generalized k-Fibonacci sequence by the method of matrix diagonalization.
\end{abstract}

Keywords: k-Fibonacci Sequence; Generalized k-Fibonacci Sequence; Binet's Formula; Diagonalization of a Matrix.

\section{Introduction}

The Fibonacci numbers have many interestiong properties and applications to almost every fields of science and art. For their amazing properties and applications one can consult $[10,17,18,19]$. The beauty of Fibonacci numbers is that they can be generalize. So these numbers can be generalized by a number of ways and these generalized forms have many interesting properties just like usual Fibonacci numbers. Many kinds of generalizations of these numbers have been presented in $[3,6,7,15]$. The two most important generalizations of Fibonacci numbers are k-Fibonacci numbers $\left\{F_{k, n}\right\}$ and k-Lucas numbers $\left\{L_{k, n}\right\}$ and these are defined as

Definition 1. For any integer $k \geq 1$, the kth Fibonacci sequence, say $\left\{F_{k, n}\right\}$ is defined recurrently by:

$$
F_{n+1}=k F_{k, n}+F_{k, n-1} \text { with } n \geq 0 F_{k, 0}=0, F_{k, 1}=1
$$

Definition 2. For any integer $k \geq 1$, the $k$ th Lucas sequence, say $\left\{L_{k, n}\right\}$ is defined recurrently by:

$$
L_{n+1}=k L_{k, n}+L_{k, n-1} \text { with } n \geq 0 L_{k, 0}=2, F_{k, 1}=k
$$

The particular cases of definition (1.1) are

- If $k=1$, we obtain the classical Fibonacci sequence $\{0,1,1,2,3,5 \ldots\}$

- If $k=2$, we obtain the Pell sequence $\{0,1,2,5,12,29 \ldots\}$

Many properties of k-Fibonacci numbers obtained directly by matrix algebra in [16]. Authors presented many interesting properties of k-Fibonacci numbers in [5, 12]. In [13] authors defined k-Fibonacci numbers by using arithmetic indexes. Wloch in [4] discussed some identities for the generalized Fibonacci numbers and the generalized Lucas numbers. In [14] author discussed some theorems or identities on the k-Lucas numbers.

Many properties of Fibonacci numbers as well as their generalizations have obtained in terms of matrices. In [8] authors studied the generalized Fibonacci and Lucas numbers by matrix methods here the authors considered two cross two matrices and after that obtained $n$th power of the matrices such as

$$
\begin{gathered}
\text { if } U(p, q)=\left[\begin{array}{rr}
p & -q \\
1 & 0
\end{array}\right] \text { and } V(p, q)=\left[\begin{array}{cc}
p^{2}-2 q & -p q \\
p & -2 q
\end{array}\right] \text { then } \\
U^{n}(p, q)=\left[\begin{array}{cc}
U_{n+1} & -q U_{n} \\
U_{n} & -q U_{n-1}
\end{array}\right] \text { and } \\
V^{n}(p, q)=\left\{\begin{array}{c}
\left(p^{2}-4 q\right)^{\frac{n}{2}}\left[\begin{array}{cc}
U_{n+1} & -q U_{n} \\
U_{n} & -q U_{n-1}
\end{array}\right] \quad \text { if } n \text { is even } \\
\left(p^{2}-4 q\right)^{\frac{n-1}{2}}\left[\begin{array}{cc}
V_{n+1} & -q V_{n} \\
V_{n} & -q V_{n-1}
\end{array}\right] \quad \text { if } n \text { is odd }
\end{array}\right.
\end{gathered}
$$

where $U_{n}$ and $V_{n}$ are nth generalized Fibonacci and Lucas numbers respectively and the authors defined these sequences recurrently by

$$
\begin{gathered}
U_{n}=p U_{n-1}-q U_{n-2}, n \geq 2, U_{0}=0, U_{1}=1 \text { and } \\
V_{n}=p V_{n-1}-q V_{n-2}, n \geq 2, V_{0}=2, V_{1}=p
\end{gathered}
$$

In [20] authors considered two cross two matrix for $U_{n}$ and $V_{n}$ in a different way and derived a number of results by using this matrix which is defined below

$$
A=\left[\begin{array}{cc}
p^{2}-2 q & p \\
-q & -2 q
\end{array}\right]
$$

In [7] author derived a number general formulas for the generalized Fibonacci sequence by matrix methods. Ahmet in [2] obtained some identities of Pell, Pell-Lucas, and Modified Pell numbers by using 
some matrix methods. Here the authors defined some two cross two matrices as

$$
N=\left[\begin{array}{ll}
3 & 1 \\
1 & 1
\end{array}\right], R=\left[\begin{array}{ll}
6 & 2 \\
2 & 2
\end{array}\right] \text { and } F=\left[\begin{array}{rr}
2 & 2 \\
2 & -2
\end{array}\right]
$$

In [1] authors derived a number of properties of k-Fibonacci and k-Lucas sequences with the help of two cross two generating matrix for these sequences, such as they proved a Binet's fomula for kFibonacci sequence and k-Lucas sequence by using the concept of diagonalization of generating matrix. So the generating matrix and its $n$th powers are given as

$$
\begin{gathered}
F=L=\left[\begin{array}{ll}
0 & 1 \\
1 & k
\end{array}\right] \text { then } F^{n}=\left[\begin{array}{cc}
F_{k, n-1} & F_{k, n+1} \\
F_{k, n} & F_{k, n-1}
\end{array}\right] \text { and } \\
L^{n}=\left[\begin{array}{cc}
\frac{2 L_{k, n}-k L_{k, n-1}}{k^{2}+4} & \frac{L_{k, n-1}+L_{k, n+1}}{k^{2}+4} \\
\frac{L_{k, n-1}+L_{k, n+1}}{k^{2}+4} & \frac{L_{k, n}+L_{k, n+2}}{k^{2}+4}
\end{array}\right]
\end{gathered}
$$

In [11] author used the same concept as in [1] and studied the k-PellLucas sequences by matrix methods.

\section{Generalized k-Fibonacci Sequence}

In the present study we find the properties of generalized k-Fibonacci sequence by matrix methods and the generalized k-Fibonacci is defined by

Definition 3. For $q, k \in N$, the generalized $k$-Fibonacci sequence, say $S_{k, n}$ is defined recurrently by:

$$
S_{n}=k S_{k, n-1}+S_{k, n-2} \text { with } n \geq 2 S_{k, 0}=q, S_{k, 1}=q k
$$

The k-Fibonacci sequence, k-Lucas sequence and generalized $\mathrm{k}$ Fibonacci sequence have the same characteristic equation $x^{2}-k x-1$. Let $r$ and $s$ the two roots of this equation. Some conspicuous points about $r$ and $s$ are

$$
\begin{aligned}
r+s=k, r s=-1, r-s & =\sqrt{k^{2}+4}, r^{2}-1=k r \\
s^{2}-1 & =k s
\end{aligned}
$$

where $r=\frac{k+\sqrt{k^{2}+4}}{2}$ and $s=\frac{k-\sqrt{k^{2}+4}}{2}$

In [14] the well-known general forms for the k-Fibonacci and kLucas sequence are known as Binets formulae are given below

$$
F_{k, n}=\frac{r^{n}-s^{n}}{r-s}, L_{k, n}=r^{n}+s^{n}
$$

and the Binet's formula for the generalized k-Fibonacci sequence (2.1) is given by

$$
S_{k, n}=q \frac{r^{n+1}-s^{n+1}}{r-s}
$$

Theorem 1. For $k \in N$, we have

$$
\begin{array}{r}
q L_{k, n}=S_{k, n}+S_{k, n-2}, n \geq 2 \\
q^{2} L_{k, n}^{2}-\left(k^{2}+4\right) S_{k, n-1}^{2}=4 q^{2}(-1)^{n}, n \geq 1
\end{array}
$$

Proof. It can be simply prove by the use of equations (2.2),(2.3) and (2.4)

\section{Generating Matrix for the Generalized k- Fibonacci Sequence}

One of the most and conventional methods for the study of the recurrences relations is generating matrix of the recurrence relations and we are aware about that Fibonacci numbers and their generalizations are the good examples of second order recurrence relations. But in the ongoing paper we concern about the generalized k-Fibonacci sequence, so generalized k-Fibonacci sequence is defined recursively as a linear combination of the $p$ terms

$a_{n+p}=c_{p-1} a_{n+p-1}+c_{p-2} a_{n+p-2}+\cdots+c_{1} a_{n+1}+c_{0} a_{n}$

where $c_{0}, c_{1} \cdots c_{p-1}$ are real constants and for detailed illustration about the generating matrix one can see [11]. If we put $p=2$ in (3.1) we get $a_{n+2}=c_{1} a_{n+1}+c_{0} a_{n}$ and after that if we recall recurrence (2.1) and take $c_{0}=1$ and $c_{1}=k$ then the matrix associated called generating matrix is given by

$$
S=\left[\begin{array}{ll}
k & 1 \\
1 & 0
\end{array}\right]
$$

Clearly $\left|S^{n}\right|=(-1)^{n}$. For the $n$th power of $S$ we have the following result

Theorem 2. For $k \in N$, we have

$$
S^{n}=q^{-1}\left[\begin{array}{cc}
S_{k, n} & S_{k, n-1} \\
S_{k, n-1} & S_{k, n-2}
\end{array}\right], n \geq 2
$$

Proof. To prove the result we will use induction on $n$. Clearly (3.3) is true for $n=2$. Suppose (3.3) is true for $n$, we get

$$
\begin{aligned}
S^{n+1} & =S^{n} S \\
& =q^{-1}\left[\begin{array}{cc}
S_{k, n} & S_{k, n-1} \\
S_{k, n-1} & S_{k, n-2}
\end{array}\right]\left[\begin{array}{cc}
k & 1 \\
1 & 0
\end{array}\right] \\
& =q^{-1}\left[\begin{array}{cc}
k S_{k, n}+S_{k, n-1} & S_{k, n} \\
k S_{k, n-1}+S_{k, n-2} & S_{k, n-1}
\end{array}\right] \\
& =q^{-1}\left[\begin{array}{cc}
S_{k, n+1} & S_{k, n} \\
S_{k, n} & S_{k, n-1}
\end{array}\right]
\end{aligned}
$$

Corollary 1. For $k, n \in N$, we have

$$
S^{n}=\left[\begin{array}{cc}
F_{k, n+1} & F_{k, n} \\
F_{k, n} & F_{k, n-1}
\end{array}\right]
$$

Theorem 3. (Cassini's Identity) For $k, n \in N$, we have

$$
S_{k, n+1} S_{k, n-1}-S_{k, n}^{2}=q^{2}(-1)^{n+1}
$$

Proof. 1

It can be simply proved by using the concept of determinats to matrices $S$ and $S^{n}$ in equations (3.2) and (3.3).

Proof. 2

Here we can employ cramers rule in [9] for $2 \times 2$ linear systems of equations to derive Cassini's identity. Consider a $2 \times 2$ linear system

$$
\begin{aligned}
& S_{k, n} a+S_{k, n-1} b=S_{k, n+1} \\
& S_{k, n+1} a+S_{k, n} b=S_{k, n+2}
\end{aligned}
$$

Clearly $S_{k, n}^{2}-S_{k, n+1} S_{k, n-1} \neq 0$ for $n \geq 1$.

Let $D=S_{k, n}^{2}-S_{k, n+1} S_{k, n-1}$ then by Cramer's rule, we get

$$
a=\frac{\left|\begin{array}{ll}
S_{k, n+1} & S_{k, n-1} \\
S_{k, n+2} & S_{k, n+1}
\end{array}\right|}{D} \text { and } b=\frac{\left|\begin{array}{cc}
S_{k, n} & S_{k, n+1} \\
S_{k, n+1} & S_{k, n+2}
\end{array}\right|}{D}
$$


By virtue of the recurrence relation (2.1), $a=k$ and $b=1$ is the unique solution of the system given in (3.6). Therefore by Cramer's rule, we get

$$
\begin{aligned}
1=\frac{\left|\begin{array}{cc}
S_{k, n} & S_{k, n+1} \\
S_{k, n+1} & S_{k, n+2}
\end{array}\right|}{D} & \Rightarrow S_{k, n+2} S_{k, n}-S_{k, n+1}^{2} \\
& =S_{k, n}^{2}-S_{k, n+1} S_{k, n-1}
\end{aligned}
$$

Let

$$
P_{k, n}=S_{k, n+1} S_{k, n-1}-S_{k, n}^{2}
$$

and

$$
P_{k, n+1}=S_{k, n+2} S_{k, n}-S_{k, n+1}^{2}
$$

Clearly $P_{k, n+1}=-P_{k, n}$ with $n \geq 1, P_{k, 1}=q^{2}$

Now the equation (3.8) is a first order linear recurrence. Thus $P_{K, n}=q^{2}(-1)^{n+1}$ is the general solution of (3.8). Hence from the equation (3.7), we get

$$
S_{k, n+1} S_{k, n-1}-S_{k, n}^{2}=q^{2}(-1)^{n+1}
$$

Theorem 4. For $k, n \in N$, we have

$$
\left[\begin{array}{c}
S_{k, n+1} \\
S_{k, n}
\end{array}\right]=S\left[\begin{array}{c}
S_{k, n} \\
S_{k, n-1}
\end{array}\right]
$$

Proof. To prove the result we will use induction on $n$. (3.9) is true for $n=1$. Suppose (3.9) is true for $n$, we get

$$
\begin{aligned}
{\left[\begin{array}{l}
S_{k, n+2} \\
S_{k, n+1}
\end{array}\right] } & =\left[\begin{array}{c}
k S_{k, n+1}+S_{k, n} \\
S_{k, n+1}
\end{array}\right] \\
& =\left[\begin{array}{ll}
k & 1 \\
1 & 0
\end{array}\right]\left[\begin{array}{c}
S_{k, n+1} \\
S_{k, n}
\end{array}\right] \\
& =\left[\begin{array}{ll}
k & 1 \\
1 & 0
\end{array}\right]\left[\begin{array}{cc}
k & 1 \\
1 & 0
\end{array}\right]\left[\begin{array}{c}
S_{k, n} \\
S_{k, n-1}
\end{array}\right] \\
& =\left[\begin{array}{ll}
k & 1 \\
1 & 0
\end{array}\right]\left[\begin{array}{c}
k S_{k, n}+S_{k, n-1} \\
S_{k, n}
\end{array}\right] \\
& =\left[\begin{array}{cc}
k & 1 \\
1 & 0
\end{array}\right]\left[\begin{array}{c}
S_{k, n+1} \\
S_{k, n}
\end{array}\right] \\
& =S\left[\begin{array}{c}
S_{k, n+1} \\
S_{k, n}
\end{array}\right]
\end{aligned}
$$

Theorem 5. For $k, n \in N$, we have

$$
\left[\begin{array}{c}
S_{k, n+1} \\
S_{k, n}
\end{array}\right]=S^{n}\left[\begin{array}{c}
S_{k, 1} \\
S_{k, 0}
\end{array}\right]
$$

Proof. It can be show simply by Principal of Mathematical Induction.

\section{Binet's Formula by Matrix Diagonalization of Generating Matrix}

In this section we will use the diagonalization of the generating matrix to obtain the Binet's fomula for the generalized k-Fibonacci sequence defined in (2.1).

Theorem 6. (Binet's Formula): For $n \geq 0$ and $k \in N$, the nth term of the generalized $k$-Fibonacci sequence is given by

$$
S_{k, n}=q \frac{r^{n+1}-s^{n+1}}{r-s}
$$

where $r$ and $s$ are the roots of the characteristic equation $x^{2}-k x-$ $1=0$
Proof. Since the generating matrix is given by $S=\left[\begin{array}{ll}k & 1 \\ 1 & 0\end{array}\right]$. Now here we have motive to diagonalize the generating matrix $S$. Since $S$ is a square matrix. So let $x$ be the eigen value of $U$ then by the Cayley Hamilton theorem on matrices, we have

$$
\begin{gathered}
|U-x I|=0 \\
\left|\begin{array}{cc}
k-x & x \\
1 & -x
\end{array}\right|=0 \\
x^{2}-k x-x=0
\end{gathered}
$$

This is the characteristic equation of the generating matrix. Let $r=\frac{k+\sqrt{k^{2}+4}}{2}$ and $s=\frac{k-\sqrt{k^{2}+4}}{2}$ are the roots of the characteristic equation and also $r$ and $s$ be the two eigen values of a square matrix $S$. Now we will try to find the eigen vectors corresponding to the eigen values $r$ and $s$. To find the eigen vectors we simply solve the system of linear equations given by

$$
(S-x I) V=0
$$

where $V$ is the column vector of order $2 \times 1$. First of all we calculate the eigen vector corresponding to the eigen value $r$ then

$$
\begin{gathered}
(S-r I) V=0 \\
{\left[\begin{array}{cc}
k-r & r \\
1 & -r
\end{array}\right]\left[\begin{array}{l}
V_{1} \\
V_{2}
\end{array}\right]=0} \\
(k-r) V_{1}+r V_{2}=0 \\
V_{1}-r V_{2}=0
\end{gathered}
$$

put $V_{2}=t$ in (4.4) we get $V_{1}=r t$. Hence the eigen vectors corresponding to $r$ are $\left[\begin{array}{c}r t \\ t\end{array}\right]$. In particular $t=1$, the eigen vector corresponding to $r$ is $\left[\begin{array}{l}r \\ 1\end{array}\right]$

Similarly the eigen vector corressponding to $s$ is $\left[\begin{array}{l}s \\ 1\end{array}\right]$. Let $A$ be the matrix of eigen vectors, so $P=\left[\begin{array}{ll}r & s \\ 1 & 1\end{array}\right]$ then $A^{-1}=$ $(r-s)^{-1}\left[\begin{array}{cc}1 & -s \\ -1 & r\end{array}\right]$. Now we consider a diagonal matrix $D$ in which eigen values of $S$ are on the main diagonal, $D=\left[\begin{array}{cc}r & 0 \\ 0 & s\end{array}\right]$. By the principal of diagonalization of matrices, we have

$$
\begin{aligned}
S & =A D A^{-1} \\
S^{n} & =\left(A D A^{-1}\right)^{n} \\
& =A D^{n} A^{-1} \\
& =(r-s)^{-1}\left[\begin{array}{ll}
r & s \\
1 & 1
\end{array}\right]\left[\begin{array}{cc}
r^{n} & 0 \\
0 & s^{n}
\end{array}\right]\left[\begin{array}{cc}
1 & -s \\
-1 & r
\end{array}\right] \\
& =(r-s)^{-1}\left[\begin{array}{cc}
r^{n+1} & s^{n+1} \\
r^{n} & s^{n}
\end{array}\right]\left[\begin{array}{cc}
1 & -s \\
-1 & r
\end{array}\right] \\
& =(r-s)^{-1}\left[\begin{array}{cc}
r^{n+1}-s^{n+1} & -s r^{n+1}+r s^{n+1} \\
r^{n}-s^{n} & -s r^{n}+r s^{n}
\end{array}\right]
\end{aligned}
$$

Since from equation (2.2) $r s=(-1)$, we have

$$
S^{n}=(r-s)^{-1}\left[\begin{array}{cc}
r^{n+1}-s^{n+1} & r^{n}-s^{n} \\
r^{n}-s^{n} & r^{n-1}-s^{n-1}
\end{array}\right]
$$

Since $\left[\begin{array}{c}S_{k, n+1} \\ S_{k, n}\end{array}\right]=S^{n}\left[\begin{array}{c}S_{k, 1} \\ S_{k, 0}\end{array}\right]=S^{n}\left[\begin{array}{c}q k \\ q\end{array}\right]=q S^{n}\left[\begin{array}{c}k \\ 1\end{array}\right]$ then 


$$
\begin{aligned}
{\left[\begin{array}{c}
S_{k, n+1} \\
S_{k, n}
\end{array}\right] } & =q(r-s)^{-1}\left[\begin{array}{cc}
r^{n+1}-s^{n+1} & r^{n}-s^{n} \\
r^{n}-s^{n} & r^{n-1}-s^{n-1}
\end{array}\right]\left[\begin{array}{l}
k \\
1
\end{array}\right] \\
& =q(r-s)^{-1}\left[\begin{array}{c}
k r^{n+1}-k s^{n+1}+r^{n}-s^{n} \\
k r^{n}-k s^{n}+r^{n-1}-s^{n-1}
\end{array}\right] \\
& =q(r-s)^{-1}\left[\begin{array}{c}
r^{n}(k r+1)-s^{n}(k s+1) \\
r^{n-1}(k r+1)-s^{n-1}(k s+1)
\end{array}\right]
\end{aligned}
$$

After using equation (2.2), we have

$$
\left[\begin{array}{c}
S_{k, n+1} \\
S_{k, n}
\end{array}\right]=\frac{q}{r-s}\left[\begin{array}{c}
r^{n+2}-s^{n+2} \\
r^{n+1}-s^{n+1}
\end{array}\right]
$$

Hence

$$
S_{k, n}=q \frac{r^{n+1}-s^{n+1}}{r-s}
$$

This is clearly the Binet's formula for generalized k-Fibonacci sequence which is defined in equation (2.4)

Theorem 7. The generalized characteristic roots of $S^{n}$ are

$$
\begin{aligned}
r^{n} & =\frac{L_{k, n}+q^{-1} \sqrt{k^{2}+4} S_{k, n-1}}{2} \\
s^{n} & =\frac{L_{k, n}-q^{-1} \sqrt{k^{2}+4} S_{k, n-1}}{2}
\end{aligned}
$$

Proof. If we write the characteristic polynomial of $S^{n}$, we have

$$
\begin{aligned}
\left|S^{n}-y I\right|= & {\left[\begin{array}{cc}
\frac{S_{k, n-2}}{q}-y & \frac{S_{k, n-1}}{q} \\
\frac{S_{k, n-1}}{q} & \frac{S_{k, n}}{q}-y
\end{array}\right] } \\
= & q^{-2}\left[\begin{array}{cc}
S_{k, n-2}-q y & S_{k, n-1} \\
S_{k, n-1} & S_{k, n}-q y
\end{array}\right] \\
= & q^{-2}\left(S_{k, n-2}-q y\right)\left(S_{k, n}-q y\right)-S_{k, n-1}^{2} \\
= & q^{-2}\left[q^{2} y^{2}-\left(S_{k, n-2}+S_{k, n}\right) q y\right. \\
& \left.+S_{k, n-2} S_{k, n}-S_{k, n-1}^{2}\right]
\end{aligned}
$$

If we recall equations (2.5) and (3.5), we have

$$
\begin{aligned}
\left|S^{n}-y I\right| & =q^{-2}\left[q^{2} y^{2}-L_{k, n} q^{2} y+q^{2}(-1)^{n}\right] \\
& =y^{2}-L_{k, n} y+(-1)^{n}
\end{aligned}
$$

Thus the characteristic equation of $S^{n}$ is

$$
y^{2}-L_{k, n} y+(-1)^{n}=0
$$

and the generalized characteristic roots are given by

$$
y=\frac{L_{k, n} \pm \sqrt{L_{k, n}^{2}-4(-1)^{n}}}{2}
$$

If we use equation (2.6) in equation (4.7), we get

$$
y=\frac{L_{k, n} \pm q^{-1} \sqrt{k^{2}+4} S_{k, n-1}}{2}
$$

Clearly the equation (4.8) has two roots and these are $r^{n}$ and $s^{n}$. Now accordingly we get the desired result as

$r^{n}=\frac{L_{k, n}+q^{-1} \sqrt{k^{2}+4} S_{k, n-1}}{2}$ and $s^{n}=\frac{L_{k, n}-q^{-1} \sqrt{k^{2}+4} S_{k, n-1}}{2}$

Since

$$
S^{n}=q^{-1}\left[\begin{array}{cc}
S_{k, n} & S_{k, n-1} \\
S_{k, n-1} & S_{k, n-2}
\end{array}\right]
$$

$$
\frac{S^{n}}{S_{k, n-2}}=q^{-1}\left[\begin{array}{cc}
\frac{S_{k, n}}{S_{k, n-2}} & \frac{S_{k, n-1}}{S_{k, n-2}} \\
\frac{S_{k, n-1}}{S_{k, n-2}} & 1
\end{array}\right]
$$

Since the ratio of the two consecutive generalized k-Fibonacci numbers is equal to $r$ that is

$$
\lim _{n \rightarrow \infty} \frac{S_{k, n-1}}{S_{k, n-2}}=r
$$

and

$$
\lim _{n \rightarrow \infty} \frac{S_{k, n-1}}{S_{k, n-2}}=\lim _{n \rightarrow \infty} \frac{S_{k, n}}{S_{k, n-1}} \frac{S_{k, n-1}}{S_{k, n-2}}=r^{2}
$$

Therefore

$$
\lim _{n \rightarrow \infty} \frac{S^{n}}{S_{k, n-2}}=q^{-1}\left[\begin{array}{cc}
r^{2} & r \\
r & 1
\end{array}\right]
$$

If we consult equation (2.2), we have

$$
\lim _{n \rightarrow \infty} \frac{S^{n}}{S_{k, n-2}}=q^{-1}\left[\begin{array}{cc}
r^{2} & r \\
r & 1
\end{array}\right]=q^{-1}\left[\begin{array}{cc}
k r+1 & r \\
r & 1
\end{array}\right]
$$

If we compute the determinants of both sides, we get the characteristic equation of the $S$ matrix as below

$$
\begin{gathered}
q^{-1}\left(k r+1-r^{2}\right)=0 \\
r^{2}-k r-1=0
\end{gathered}
$$

\section{Conclusion}

In the present study we obtained $n$th power of the matrix and some properties have been obtained for the generalized k-Fibonacci sequence by matrix methods.

\section{Acknowledgement}

we would like to thank anonymous referee for cautiously reading the paper and for their remarks which decently upgraded the paper.

\section{References}

[1] A. Borges, P. Catarino, A. P. Aires, P. Vasco and H. Campos. "Two-byTwo Matrices Involving k-Fibonacci and k-Lucas Sequences", Applied Mathematical Sciences, 8(34):1659-1666, 2014.

[2] A. Dasdemir. "On the Pell, Pell-Lucas and Modified Pell Numbers By Matrix Method”, Applied Mathematical Sciences, 5(64):3173-3181, 2011

[3] A. F. Horadam. "Generalized Fibonacci Sequences", The American Mathematical Monthly, 68(5):455-459, 1982.

[4] A. Wloch. "Some identities for the Generalized Fibonacci numbers and the Generalized Lucas numbers", Applied Mathematics and Computation, 219:5564-5568, 2013.

[5] C. Bolat. "On the Properties of k-Fibonacci numbers". Int. J. contemp. Math. Sciences, 5(22):1097-1105, 2010.

[6] C. K. Ho. and C. Y. Chong. "Odd and Even Sums of Generalized Fibonacci Numbers by Matrix Methods", AIP Conference Proceedings, 1602, 1026 (2014); doi: 10.1063/1.4882610

[7] D. Kalman. "Generalized Fibonacci numbers by Matrix Methods', The Fibonacci Quarterly, 20(1):73-76, 1982.

[8] G. C. Morales. "On Generalized Fibonacci and Lucas Numbers by Matrix Methods", Hacettepe Journal of Mathematics and Statistics, 42(2):173-179, 2013.

[9] http://mathworld.wolfram.com/CramersRule.html.

[10] N. N. Vorobyov. "The Fibonacci Numbers", D. C. Health and company Boston, 1963.

[11] P. Catarino. "A Note Involving Two-by-Two Matrices of the k-Pell and k-Pell-Lucas Sequences", International Mathematical Forum, 8(32):1561-1568, 2013

[12] P. Catarino. "On Some Identities for k-Fibonacci Sequence”, Int. J. contemp. Math. Sciences. 9(1):37-42, 2014.

[13] S. Falcon and A. Plaza. "On k-Fibonacci numbers of arithmetic indexes", Applied Mathematics and Computation,208:180-185, 2009.

[14] S. Falcon. "On the k-Lucas Numbers", Int. J. contemp. Math. Sciences. 6(21):1039-1050, 2011. 
[15] S. Falcon. "Generalized $(k, r)$ Fibonacci Numbers", Gen. Math. Notes., 25(2):148-158, 2014

[16] S. Falcon and A. Plaza. "On the Fibonacci k-numbers", Chaos, Solitons and Fractals., 32:1615-1624, 2007.

[17] S. Vajda. "Fibonacci and Lucas Numbers and the Golden Section. Theory and Applications", Ellis Horwood Limited, 1989.

[18] T. Koshy. "Fibonacci and Lucas Numbers with Applications", John Wiley and Sons, New york, 2001

[19] V. E. Hoggatt. "Fibonacci and Lucas Numbers", Houghton-Mifflin, Co., Boston, 1969.

[20] Z. Akyuz and S. Halici. "Some identities deriving from the $n$th power of a special matrix", Advances in Difference Equations, DOI: $10.1186 / 1687-1847-2012-223$ 